\title{
8-Bromo-7-hydroxyquinoline as a Photoremovable Protecting Group for Physiological Use: Mechanism and Scope
}

Yue Zhu, ${ }^{\dagger}$ Christopher M. Pavlos, ${ }^{+}$John P. Toscano, ${ }^{*}$ and Timothy M. Dore ${ }^{\dagger *}$

${ }^{\dagger}$ Department of Chemistry, University of Georgia, Athens, Georgia 30602-2556 and Department of Chemistry, Johns Hopkins University, Baltimore, Maryland 21218-2685

\section{LC-MS Data}

LC of BHQ-OAc photolysis in $\mathrm{H}_{2} \mathrm{O}$

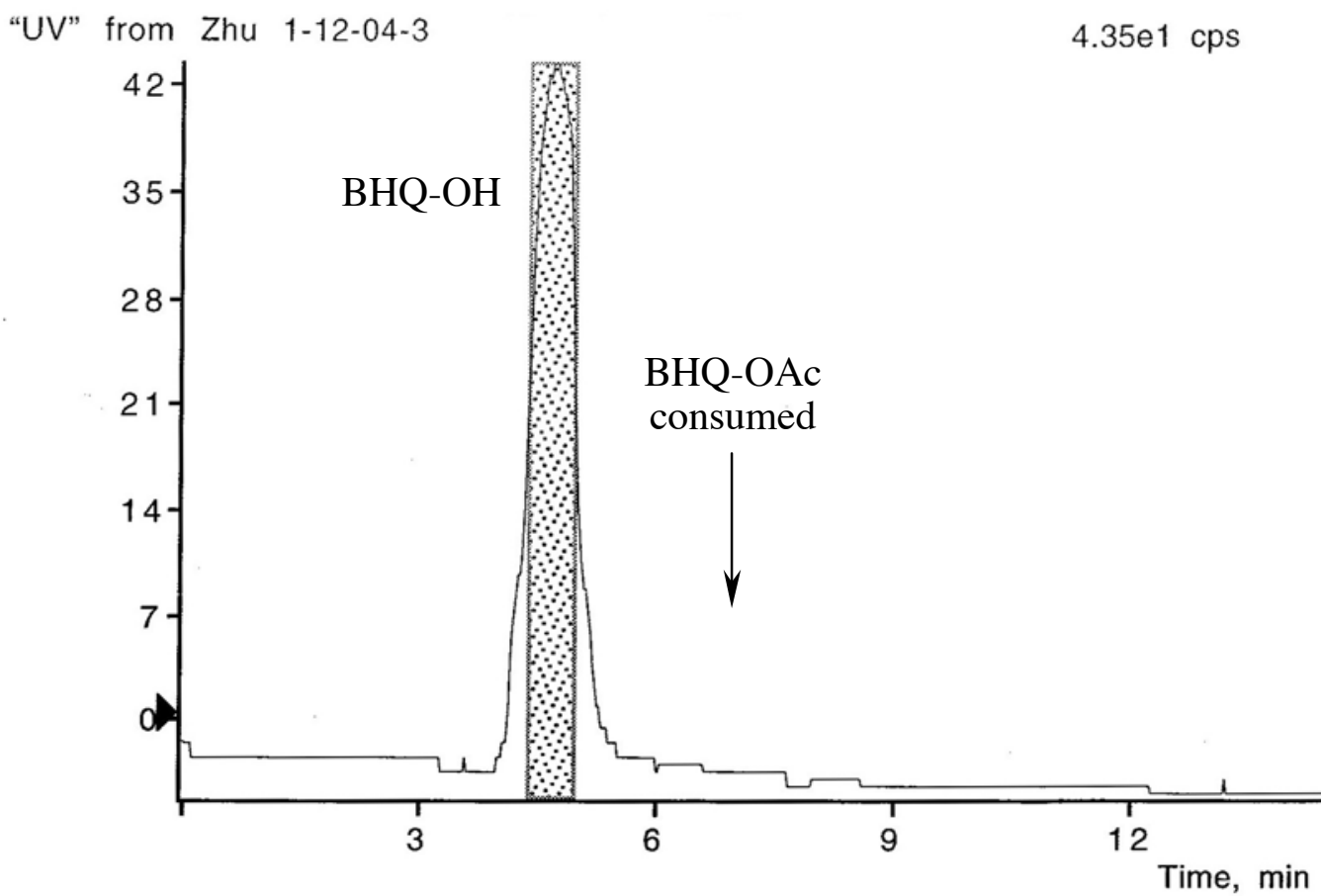

MS of highlighted peak in chromatogram above

Spectrum from $4.71 \mathrm{~min}$ (31 scans) from Zhu 1-12-04-3

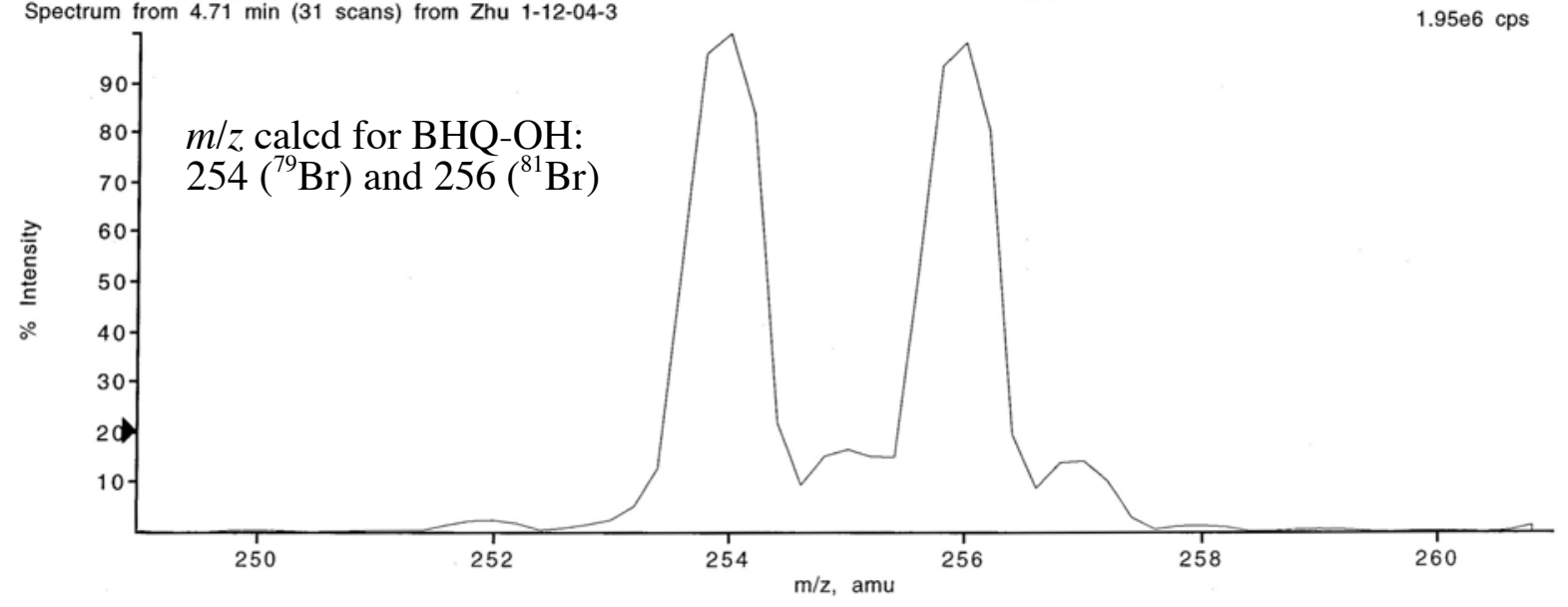


LC of BHQ-OAc photolysis in $\mathrm{H}_{2}{ }^{18} \mathrm{O}$

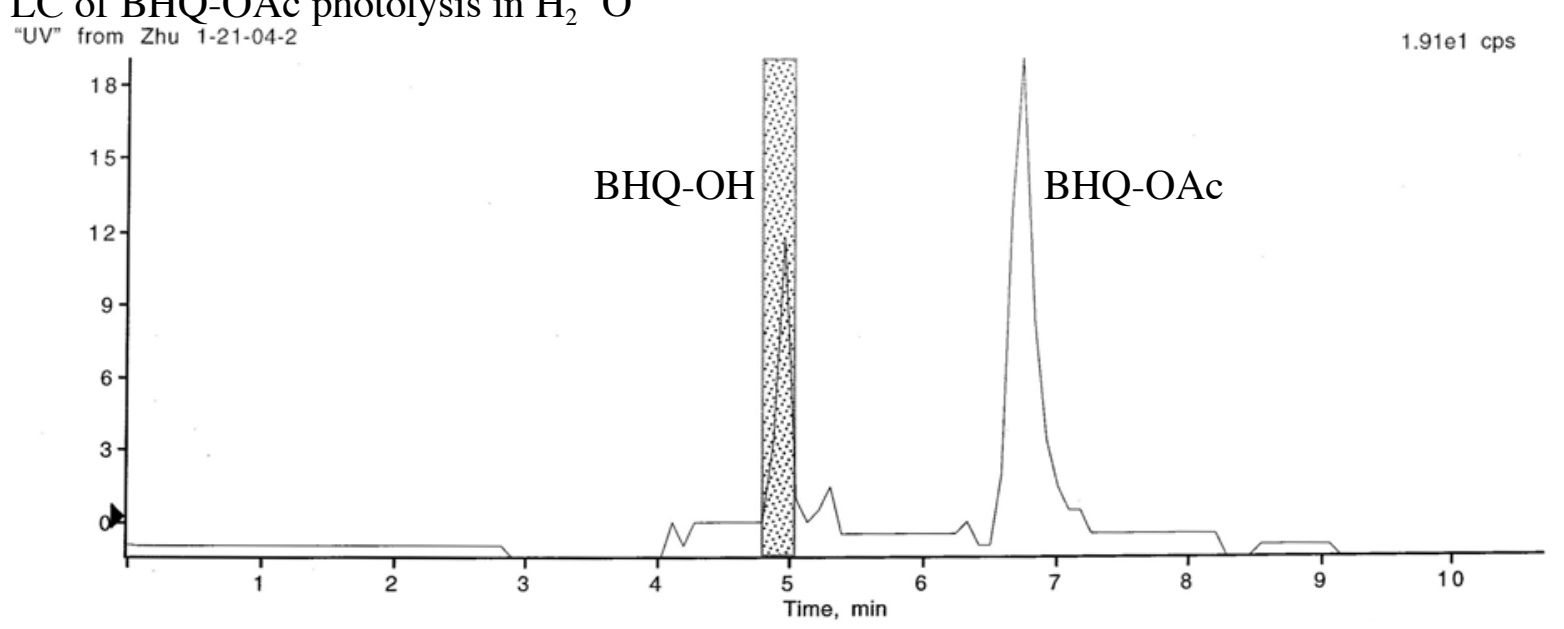

MS of highlighted peak in chromatogram above

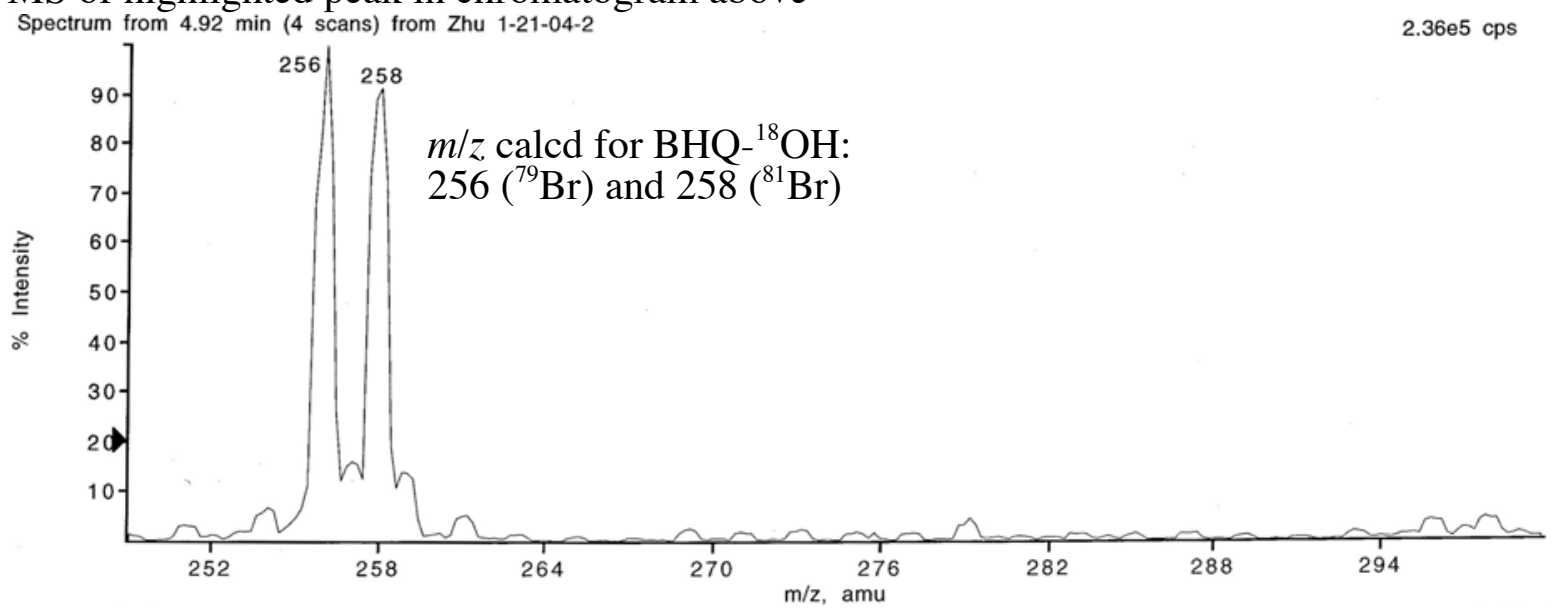

\section{Calculations}

Calculations were carried out with Gaussian 98, Revision A7: M. J. Frisch, G. W. Trucks, H. B. Schlegel, G. E. Scuseria,M. A. Robb, J. R. Cheeseman, V. G. Zakrzewski, J. A. Montgomery, Jr., R. E. Stratmann, J. C. Burant, S. Dapprich, J. M. Millam, A. D. Daniels, K. N. Kudin, M. C. Strain, O. Farkas, J. Tomasi, V. Barone, M. Cossi, R. Cammi, B. Mennucci, C. Pomelli, C. Adamo, S. Clifford, J. Ochterski, G. A. Petersson, P. Y. Ayala, Q. Cui, K. Morokuma, D. K. Malick, A. D. Rabuck, K. Raghavachari, J. B. Foresman, J. Cioslowski, J. V. Ortiz, A. G. Baboul, B. B. Stefanov, G. Liu, A. Liashenko, P. Piskorz, I. Komaromi, R. Gomperts, R. L. Martin, D. J. Fox, T. Keith, M. A. Al-Laham, C. Y. Peng, A. Nanayakkara, C. Gonzalez, M. Challacombe, P. M. W. Gill, B. Johnson, W. Chen, M. W. Wong, J. L. Andres, C. Gonzalez, M. Head-Gordon, E. S. Replogle, and J. A. Pople, Gaussian, Inc., Pittsburgh PA, 1998. 
B3LYP/6-31G* Optimized Geometry for BHQ-OAc Lowest Triplet State

\begin{tabular}{|c|c|c|c|c|}
\hline \multirow{2}{*}{$\begin{array}{c}\text { Center } \\
\text { Number }\end{array}$} & \multirow{2}{*}{$\begin{array}{l}\text { Atomic } \\
\text { Number }\end{array}$} & \multicolumn{3}{|c|}{$\begin{array}{l}\text { Coordinates } \\
\text { (Angstroms) }\end{array}$} \\
\hline & & $\mathbf{X}$ & $\mathbf{Y}$ & $\mathbf{Z}$ \\
\hline 1 & 6 & -3.366623 & 2.023088 & -0.044263 \\
\hline 2 & 6 & -3.407956 & 0.681808 & 0.20183 \\
\hline 3 & 6 & -2.141685 & -0.075997 & 0.217902 \\
\hline 4 & 6 & -0.8724 & 0.546447 & -0.015494 \\
\hline 5 & 6 & -0.875281 & 1.968002 & -0.271864 \\
\hline 6 & 6 & -2.116688 & 2.670348 & -0.2803 \\
\hline 7 & 7 & 0.233284 & -0.198493 & 0.013572 \\
\hline 8 & 6 & 1.440605 & 0.414618 & -0.212233 \\
\hline 9 & 6 & 1.548567 & 1.760876 & -0.465581 \\
\hline 10 & 6 & 0.37137 & 2.55935 & -0.497492 \\
\hline 11 & 6 & 2.602156 & -0.540528 & -0.150546 \\
\hline 12 & 8 & 3.823907 & 0.176347 & -0.39644 \\
\hline 13 & 6 & 4.958446 & -0.564859 & -0.370574 \\
\hline 14 & 6 & 6.168067 & 0.298898 & -0.642072 \\
\hline 15 & 8 & 4.974012 & -1.755997 & -0.157014 \\
\hline 16 & 35 & -2.22563 & -1.922999 & 0.559347 \\
\hline 17 & 8 & -4.580797 & 0.053806 & 0.427043 \\
\hline 18 & 1 & -4.293401 & 2.586027 & -0.057526 \\
\hline 19 & 1 & -2.10551 & 3.738598 & -0.474228 \\
\hline 20 & 1 & 2.521422 & 2.205693 & -0.638649 \\
\hline 21 & 1 & 0.438102 & 3.625077 & -0.696261 \\
\hline 22 & 1 & 2.487482 & -1.339369 & -0.892678 \\
\hline 23 & 1 & 2.651725 & -1.02838 & 0.830064 \\
\hline 24 & 1 & 7.066664 & -0.318238 & -0.616355 \\
\hline 25 & 1 & 6.240783 & 1.093155 & 0.108236 \\
\hline 26 & 1 & 6.075736 & 0.780774 & -1.621033 \\
\hline 27 & 1 & -4.40741 & -0.894466 & 0.581696 \\
\hline
\end{tabular}

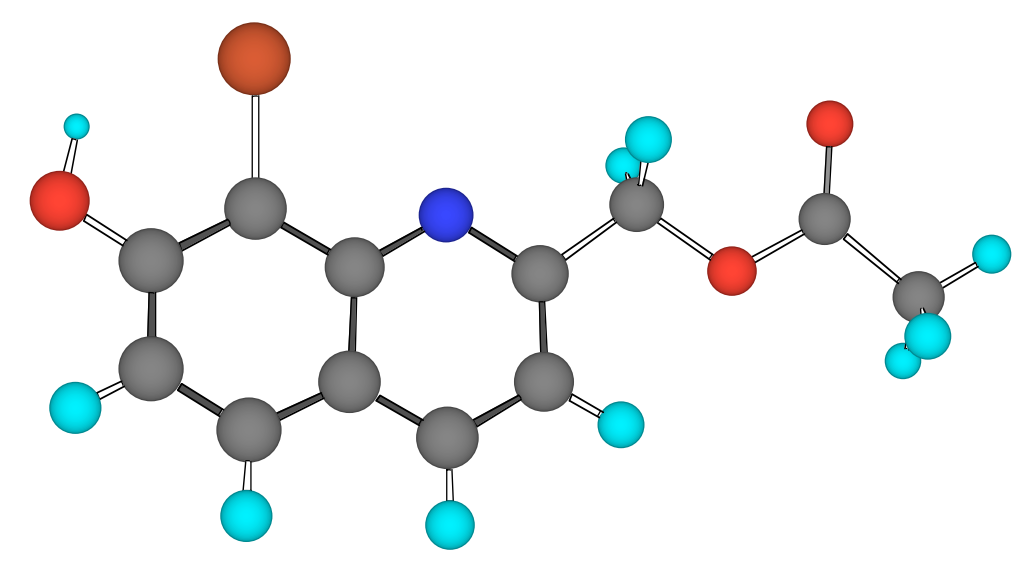


B3LYP/6-31G* Computed Vibrational Frequencies for the Lowest Triplet State of BHQ-OAc

\begin{tabular}{|c|c|c|c|c|}
\hline Calculated & Scaled by 0.96 & Intensity & Observed & Mode \\
\hline 19.5 & 18.7 & 2.1 & - & - \\
\hline 27.7 & 26.6 & 0.3 & - & - \\
\hline 53.4 & 51.2 & 0.0 & - & - \\
\hline 59.2 & 56.8 & 0.3 & - & - \\
\hline 63.4 & 60.9 & 0.5 & - & - \\
\hline 112.4 & 107.9 & 0.1 & - & - \\
\hline 130.3 & 125.1 & 1.4 & - & - \\
\hline 139.9 & 134.3 & 0.4 & - & - \\
\hline 183.0 & 175.7 & 8.0 & - & - \\
\hline 218.1 & 209.4 & 4.6 & - & - \\
\hline 256.8 & 246.5 & 1.5 & - & - \\
\hline 264.2 & 253.6 & 2.6 & - & - \\
\hline 265.1 & 254.5 & 2.3 & - & - \\
\hline 298.1 & 286.2 & 1.9 & - & - \\
\hline 362.5 & 348.0 & 0.0 & - & - \\
\hline 381.2 & 366.0 & 7.8 & - & - \\
\hline 411.4 & 394.9 & 2.8 & - & - \\
\hline 437.8 & 420.2 & 82.7 & - & - \\
\hline 463.8 & 445.3 & 31.3 & - & - \\
\hline 466.3 & 447.6 & 13.1 & - & - \\
\hline 530.5 & 509.3 & 8.9 & - & - \\
\hline 541.4 & 519.7 & 2.8 & - & - \\
\hline 544.4 & 522.6 & 3.2 & - & - \\
\hline 597.2 & 573.3 & 1.1 & - & - \\
\hline 603.6 & 579.4 & 3.9 & - & - \\
\hline 632.8 & 607.5 & 12.8 & - & - \\
\hline 648.3 & 622.4 & 12.0 & - & - \\
\hline 730.3 & 701.0 & 20.0 & - & - \\
\hline 733.0 & 703.7 & 9.6 & - & - \\
\hline 777.1 & 746.1 & 19.5 & - & - \\
\hline 778.8 & 747.7 & 8.6 & - & - \\
\hline 816.3 & 783.6 & 25.1 & - & - \\
\hline 838.8 & 805.2 & 17.0 & - & - \\
\hline 861.0 & 826.6 & 5.3 & - & - \\
\hline 901.6 & 865.6 & 0.2 & - & - \\
\hline 939.9 & 902.3 & 67.8 & - & - \\
\hline 958.2 & 919.9 & 23.5 & - & - \\
\hline 991.3 & 951.6 & 0.8 & - & - \\
\hline 1016.7 & 976.0 & 24.3 & - & - \\
\hline 1031.6 & 990.3 & 0.0 & - & - \\
\hline 1042.8 & 1001.0 & 2.8 & - & - \\
\hline 1076.8 & 1033.7 & 7.6 & - & - \\
\hline 1092.7 & 1049.0 & 156.6 & - & - \\
\hline
\end{tabular}




\begin{tabular}{|c|c|c|c|c|}
\hline Calculated & Scaled by 0.96 & Intensity & Observed & Mode \\
\hline 1101.9 & 1057.8 & 9.8 & - & - \\
\hline 1127.0 & 1081.9 & 15.8 & - & - \\
\hline 1238.7 & 1189.2 & 44.8 & - & - \\
\hline 1249.3 & 1199.3 & 1.8 & - & - \\
\hline 1262.6 & 1212.1 & 5.3 & - & - \\
\hline 1265.6 & 1214.9 & 334.4 & - & - \\
\hline 1284.4 & 1233.0 & 567.6 & - & - \\
\hline 1328.7 & 1275.5 & 61.7 & - & - \\
\hline 1356.0 & 1301.8 & 9.6 & - & - \\
\hline 1367.7 & 1313.0 & 45.2 & - & - \\
\hline 1396.1 & 1340.3 & 14.9 & - & - \\
\hline 1423.5 & 1366.6 & 25.5 & - & - \\
\hline 1429.1 & 1371.9 & 236.8 & 1404 & Aromatic C-H Bend \\
\hline 1445.5 & 1387.7 & 14.2 & 1404 & Aromatic C-H Bend \\
\hline 1479.6 & 1420.5 & 4.9 & 1404 & Aromatic C-H Bend \\
\hline 1500.0 & 1440.0 & 62.6 & 1404 & Aromatic C-H Bend \\
\hline 1501.2 & 1441.2 & 16.6 & - & - \\
\hline 1506.0 & 1445.7 & 8.1 & - & - \\
\hline 1521.0 & 1460.1 & 17.4 & - & - \\
\hline 1597.2 & 1533.3 & 113.8 & 1532 & Aromatic $C=C$ Stretch \\
\hline 1660.6 & 1594.2 & 67.4 & 1572 & Aromatic $C=C$ Stretch \\
\hline 1836.3 & 1762.8 & 237.2 & 1736 & Carbonyl Stretch \\
\hline 3057.4 & 2935.1 & 7.6 & - & - \\
\hline 3071.0 & 2948.2 & 2.5 & - & - \\
\hline 3092.5 & 2968.8 & 15.8 & - & - \\
\hline 3133.1 & 3007.8 & 6.6 & - & - \\
\hline 3183.2 & 3055.8 & 9.4 & - & - \\
\hline 3198.0 & 3070.0 & 8.5 & - & - \\
\hline 3204.5 & 3076.3 & 23.7 & - & - \\
\hline 3227.7 & 3098.6 & 4.6 & - & - \\
\hline 3237.5 & 3108.0 & 8.5 & - & - \\
\hline 3631.0 & 3485.8 & 110.2 & - & - \\
\hline
\end{tabular}

Observed Product Stretching Frequencies

TRIR Observed Products

\begin{tabular}{ccc}
\hline sserved Products & Acetate & BHQ-OH \\
\hline 1316 & - & 1294 \\
1360 & 1339 & 1346 \\
1412 & 1413 & 1434 \\
$1484^{*}$ & - & - \\
1542 & 1563 & 1559 \\
$1584^{*}$ & 1563 & 1559 \\
$1644^{*}$ & 1640 & 1618 \\
$1768^{*}$ & 1705 & 1730 \\
\hline
\end{tabular}

* Denotes peaks overlapping with ground-state depletion. 
B3LYP/6-31G* Optimized Geometry for BHQ-phosphate Lowest Triplet State

\begin{tabular}{ccccc}
\hline $\begin{array}{c}\text { Center } \\
\text { Number }\end{array}$ & $\begin{array}{c}\text { Atomic } \\
\text { Number }\end{array}$ & $\mathbf{X}$ & $\begin{array}{c}\text { Coordinates } \\
\text { (Angstroms) }\end{array}$ \\
\hline \hline 1 & 6 & 2.657216 & -3.776997 & $\mathbf{Z}$ \\
2 & 6 & 3.227214 & -2.571794 & 1.54309 \\
3 & 6 & 2.351524 & -1.41341 & 0.997174 \\
4 & 6 & 0.920687 & -1.507917 & 1.049123 \\
5 & 6 & 0.361367 & -2.803119 & 1.363179 \\
6 & 6 & 1.236837 & -3.899015 & 1.602814 \\
7 & 7 & 0.19072 & -0.419868 & 0.814204 \\
8 & 6 & -1.179834 & -0.529226 & 0.862466 \\
9 & 6 & -1.80825 & -1.719447 & 1.151661 \\
10 & 6 & -1.036733 & -2.881237 & 1.413549 \\
11 & 35 & 3.159172 & 0.233773 & 0.598116 \\
12 & 8 & 4.566489 & -2.437292 & 1.187198 \\
13 & 6 & -1.93798 & 0.72843 & 0.568593 \\
14 & 8 & -1.89673 & 0.972468 & -0.868091 \\
15 & 15 & -2.339215 & 2.409926 & -1.429088 \\
16 & 8 & -3.954495 & 2.292638 & -1.311181 \\
17 & 8 & -2.000022 & 2.265539 & -2.990227 \\
18 & 8 & -1.761882 & 3.621539 & -0.815403 \\
19 & 6 & -4.73255 & 3.500101 & -1.401835 \\
20 & 1 & -2.410986 & 1.108664 & -3.738365 \\
21 & 1 & 3.296275 & -4.632781 & 1.729603 \\
22 & 1 & -3.489272 & 0.946823 & -3.641425 \\
23 & 1 & -1.87166 & 0.223039 & -3.392258 \\
24 & 1 & -2.893098 & -1.771875 & 1.178139 \\
25 & 1 & -1.521127 & -3.824485 & 1.647786 \\
26 & 1 & -1.481254 & -1.51023 & 0.966241 \\
27 & 1 & -2.985562 & 0.63903 & 0.875139 \\
28 & -5.754965 & 3.228786 & -1.13188 \\
29 & -4.716072 & 3.892211 & -2.424606 \\
30 & -4.346752 & 4.257525 & -0.714901 \\
31 & 1 & & &
\end{tabular}




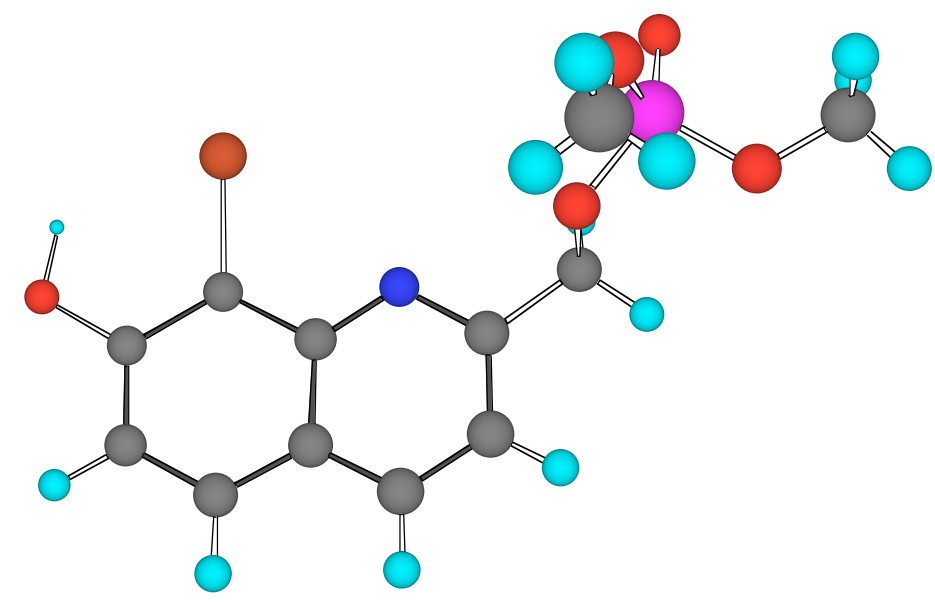

B3LYP/6-31G* Computed Vibrational Frequencies for the Lowest Triplet State of BHQ-phosphate

\begin{tabular}{|c|c|c|c|c|}
\hline Calculated & Scaled by 0.96 & Intensity & Observed & Mode \\
\hline 8.2 & 7.9 & 1.2 & - & - \\
\hline 10.3 & 9.9 & 1.4 & - & - \\
\hline 28.3 & 27.2 & 0.0 & - & - \\
\hline 50.7 & 48.7 & 2.0 & - & - \\
\hline 59.5 & 57.1 & 0.8 & - & - \\
\hline 76.6 & 73.5 & 3.4 & - & - \\
\hline 86.8 & 83.3 & 2.5 & - & - \\
\hline 106.0 & 101.8 & 0.6 & - & - \\
\hline 119.0 & 114.3 & 0.3 & - & - \\
\hline 127.1 & 122.0 & 3.8 & - & - \\
\hline 147.3 & 141.4 & 0.5 & - & - \\
\hline 156.7 & 150.5 & 2.6 & - & - \\
\hline 160.8 & 154.3 & 3.7 & - & - \\
\hline 210.9 & 202.5 & 1.2 & - & - \\
\hline 224.2 & 215.2 & 9.3 & - & - \\
\hline 258.0 & 247.7 & 0.6 & - & - \\
\hline 279.4 & 268.2 & 1.1 & - & - \\
\hline 289.6 & 278.0 & 0.8 & - & - \\
\hline 303.9 & 291.7 & 2.1 & - & - \\
\hline 341.8 & 328.1 & 1.3 & - & - \\
\hline 356.3 & 342.0 & 28.4 & - & - \\
\hline 384.0 & 368.7 & 4.6 & - & - \\
\hline 390.7 & 375.0 & 3.0 & - & - \\
\hline 429.3 & 412.1 & 27.3 & - & - \\
\hline 436.4 & 419.0 & 11.3 & - & - \\
\hline 452.2 & 434.1 & 58.5 & - & - \\
\hline 468.9 & 450.2 & 36.6 & - & - \\
\hline 476.4 & 457.3 & 66.8 & - & - \\
\hline 503.5 & 483.4 & 20.8 & - & - \\
\hline
\end{tabular}




\begin{tabular}{|c|c|c|c|c|}
\hline Calculated & Scaled by 0.96 & Intensity & Observed & Mode \\
\hline "518.6 & $4 \quad 497.8$ & 111.4 & - & - \\
\hline 538.7 & 517.1 & 13.0 & - & - \\
\hline 581.0 & 557.8 & 3.9 & - & - \\
\hline 634.1 & 608.8 & 2.6 & - & - \\
\hline 646.5 & 620.6 & 13.7 & - & - \\
\hline 717.7 & 689.0 & 46.5 & - & - \\
\hline 740.4 & 710.8 & 21.4 & - & - \\
\hline 743.2 & 713.5 & 1.7 & - & - \\
\hline 776.0 & 745.0 & 17.6 & - & - \\
\hline 800.4 & 768.4 & 28.0 & - & - \\
\hline 816.7 & 784.0 & 32.4 & - & - \\
\hline 830.7 & 797.4 & 85.0 & - & - \\
\hline 841.2 & 807.6 & 95.1 & - & - \\
\hline 861.1 & 826.6 & 10.6 & - & - \\
\hline 876.6 & 841.6 & 133.2 & - & - \\
\hline 915.8 & 879.2 & 35.5 & - & - \\
\hline 948.6 & 910.7 & 87.6 & - & - \\
\hline 988.1 & 948.6 & 2.9 & - & - \\
\hline 1010.0 & 969.6 & 886.4 & - & - \\
\hline 1037.6 & 996.1 & 74.6 & - & - \\
\hline 1068.6 & 1025.9 & 32.6 & - & - \\
\hline 1077.8 & 1034.7 & 284.6 & - & - \\
\hline 1096.1 & 1052.2 & 115.7 & - & - \\
\hline 1109.7 & 1065.3 & 11.5 & - & - \\
\hline 1148.6 & 1102.7 & 22.0 & - & - \\
\hline 1188.2 & 1140.7 & 0.3 & - & - \\
\hline 1189.7 & 1142.1 & 0.8 & 1404 & - \\
\hline 1209.6 & 1161.2 & 15.6 & 1404 & - \\
\hline 1212.1 & 1163.6 & 14.9 & 1404 & - \\
\hline 1237.2 & 1187.7 & 12.4 & 1404 & - \\
\hline 1262.0 & 1211.5 & 22.6 & - & - \\
\hline 1265.3 & 1214.7 & 125.0 & - & - \\
\hline 1277.6 & 1226.5 & 28.5 & - & - \\
\hline 1312.1 & 1259.6 & 218.0 & - & - \\
\hline 1340.6 & 1287.0 & 64.9 & - & - \\
\hline 1363.7 & 1309.1 & 25.7 & - & - \\
\hline 1375.8 & 1320.8 & 40.3 & - & - \\
\hline 1417.4 & 1360.7 & 29.5 & 1352 & $\mathrm{CH} 2$ Bend \\
\hline 1426.4 & 1369.3 & 15.4 & - & - \\
\hline 1435.2 & 1377.8 & 246.6 & 1384 & Aromatic C-H Bend \\
\hline 1483.1 & 1423.8 & 14.3 & 1420 & Aromatic C-H Bend \\
\hline 1497.3 & 1437.4 & 44.9 & 1420 & Aromatic $\mathrm{C}-\mathrm{H}$ Bend \\
\hline 1497.5 & 1437.6 & 54.1 & 1420 & Aromatic $\mathrm{C}-\mathrm{H}$ Bend \\
\hline 1499.7 & 1439.7 & 0.7 & 1420 & Aromatic C-H Bend \\
\hline 1522.0 & 1461.1 & 3.8 & - & - \\
\hline
\end{tabular}




\begin{tabular}{ccccc}
\hline Calculated & Scaled by 0.96 & Intensity & Observed & Mode \\
\hline \hline 1523.9 & 1462.9 & 3.2 & - & - \\
1525.9 & 1464.8 & 1.0 & - & - \\
1533.2 & 1471.9 & 7.3 & - & - \\
1535.7 & 1474.2 & 6.4 & - & - \\
1594.6 & 1530.8 & 46.7 & 1528 & Aromatic C=C Stretch \\
1654.6 & 1588.4 & 96.3 & 1568 & Aromatic C=C Stretch \\
3060.4 & 2938.0 & 55.5 & - & - \\
3065.5 & 2942.9 & 37.7 & - & - \\
3073.1 & 2950.1 & 21.2 & - & - \\
3130.0 & 3004.8 & 8.0 & - & - \\
3138.4 & 3012.9 & 24.9 & - & - \\
3143.7 & 3017.9 & 19.3 & - & - \\
3166.5 & 3039.8 & 15.7 & - & - \\
3166.8 & 3040.2 & 22.6 & - & - \\
3194.3 & 3066.5 & 19.1 & - & - \\
3202.1 & 3074.0 & 7.8 & - & - \\
3209.6 & 3081.2 & 24.8 & - & - \\
3229.7 & 3100.5 & 3.7 & - & - \\
3621.4 & 3476.5 & 109.3 & - & \\
\hline
\end{tabular}

Observed Product Stretching Frequencies

\begin{tabular}{ccc}
\hline TRIR Observed Products & NaOPO $(\mathbf{O M e})_{2}$ & BHQ-OH \\
\hline \hline 1412 & - & 1434 \\
1484 & 1466 & - \\
1542 & & 1559 \\
$1576^{*}$ & & 1559 \\
\hline
\end{tabular}

*Denotes peaks overlapping with ground-state depletion. 


\section{${ }^{1}$ H NMR Data}

TBDPS-HQ-benzoate (2)
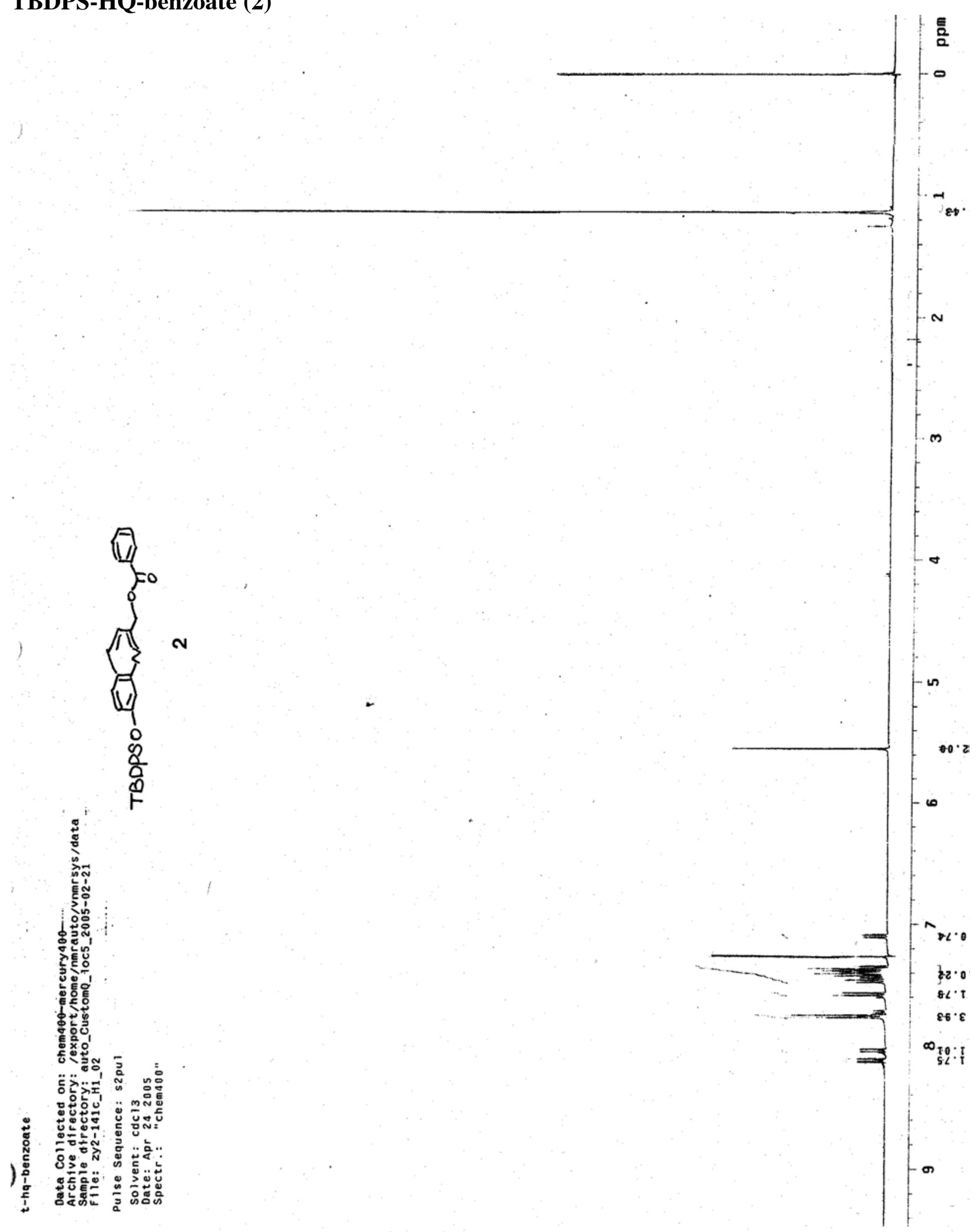


\section{TBDPS-BHQ-benzoate (3)}

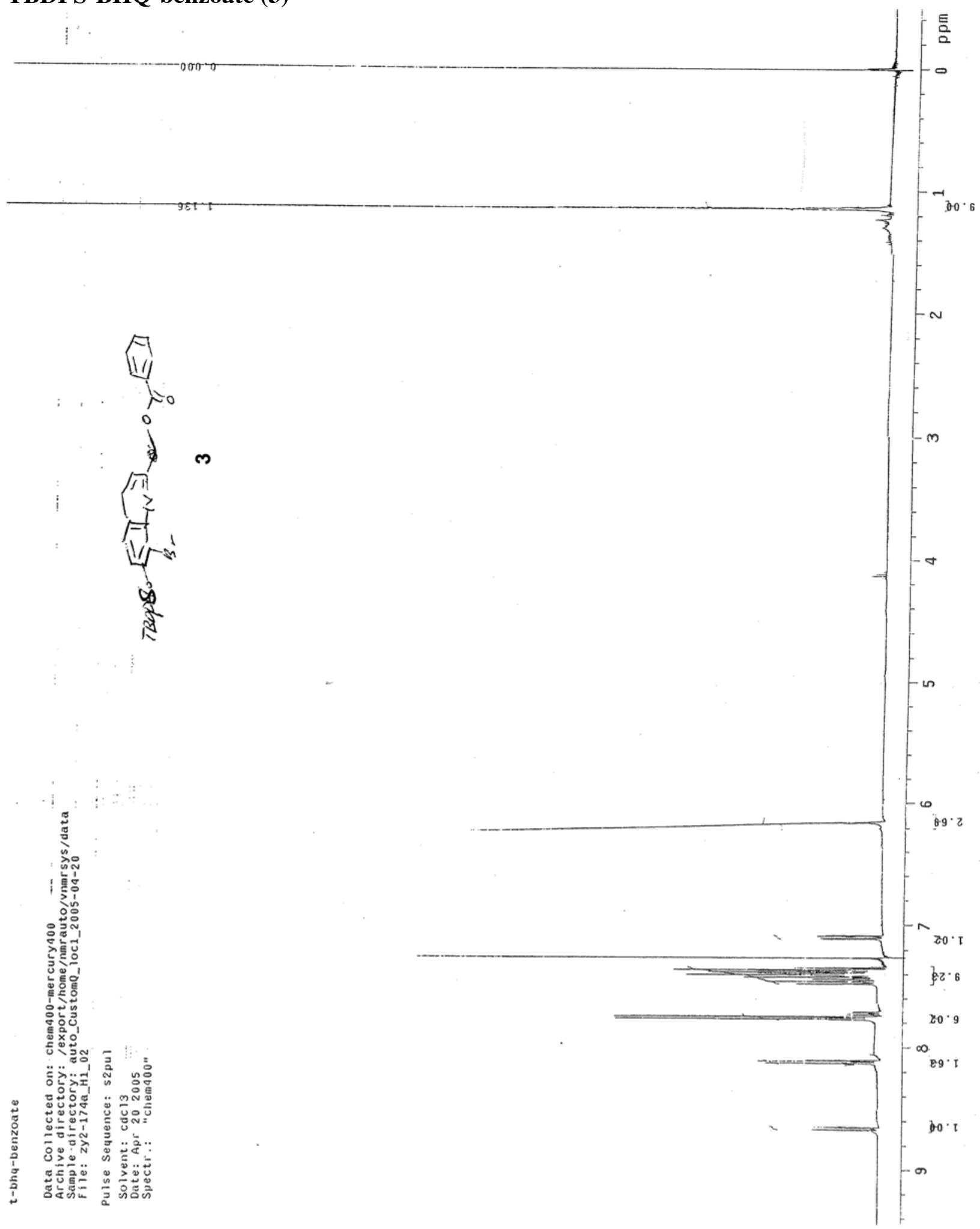




\section{BHQ-benzoate (4)}

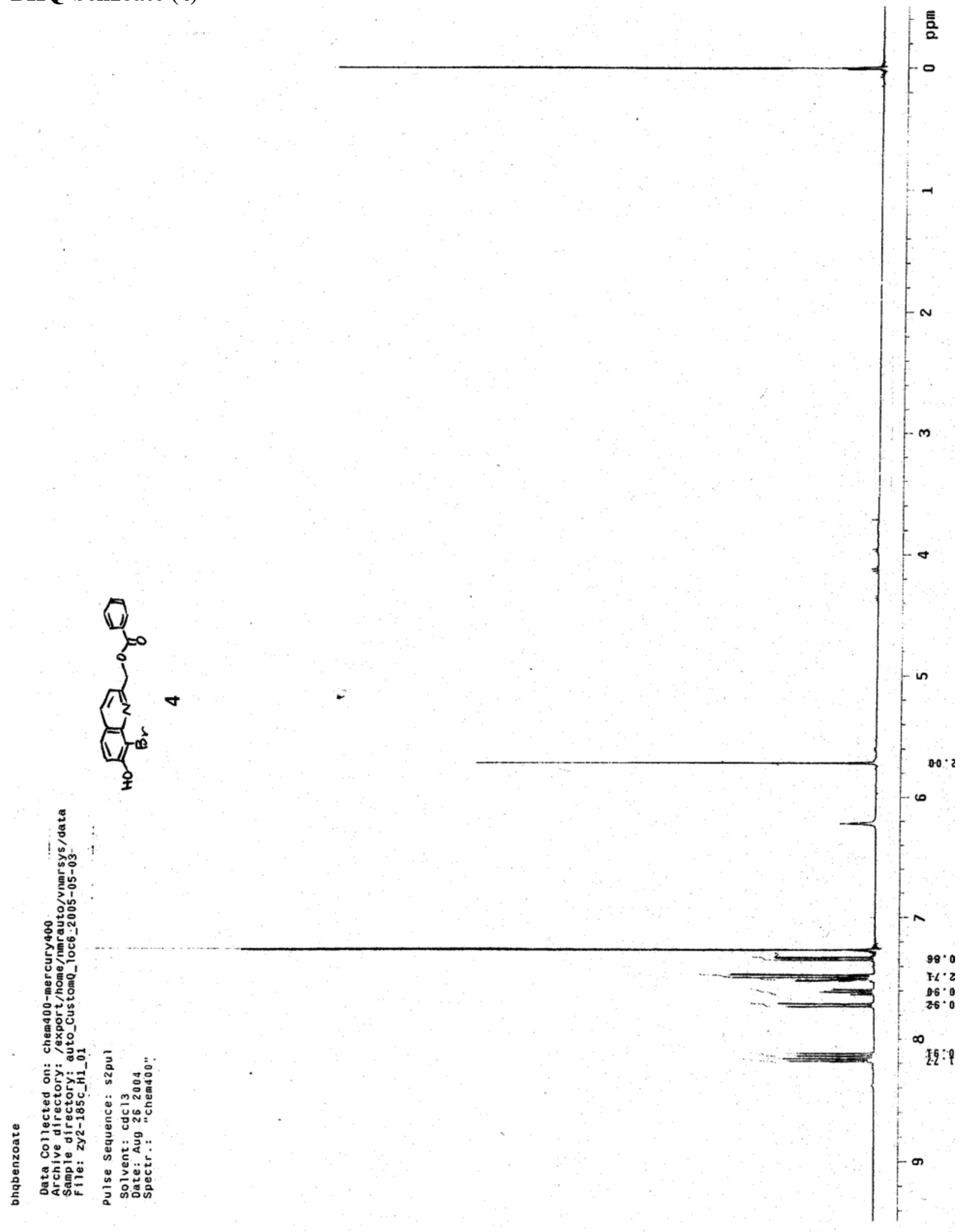




\section{TBDPS-HQ-piperonylate (5)}

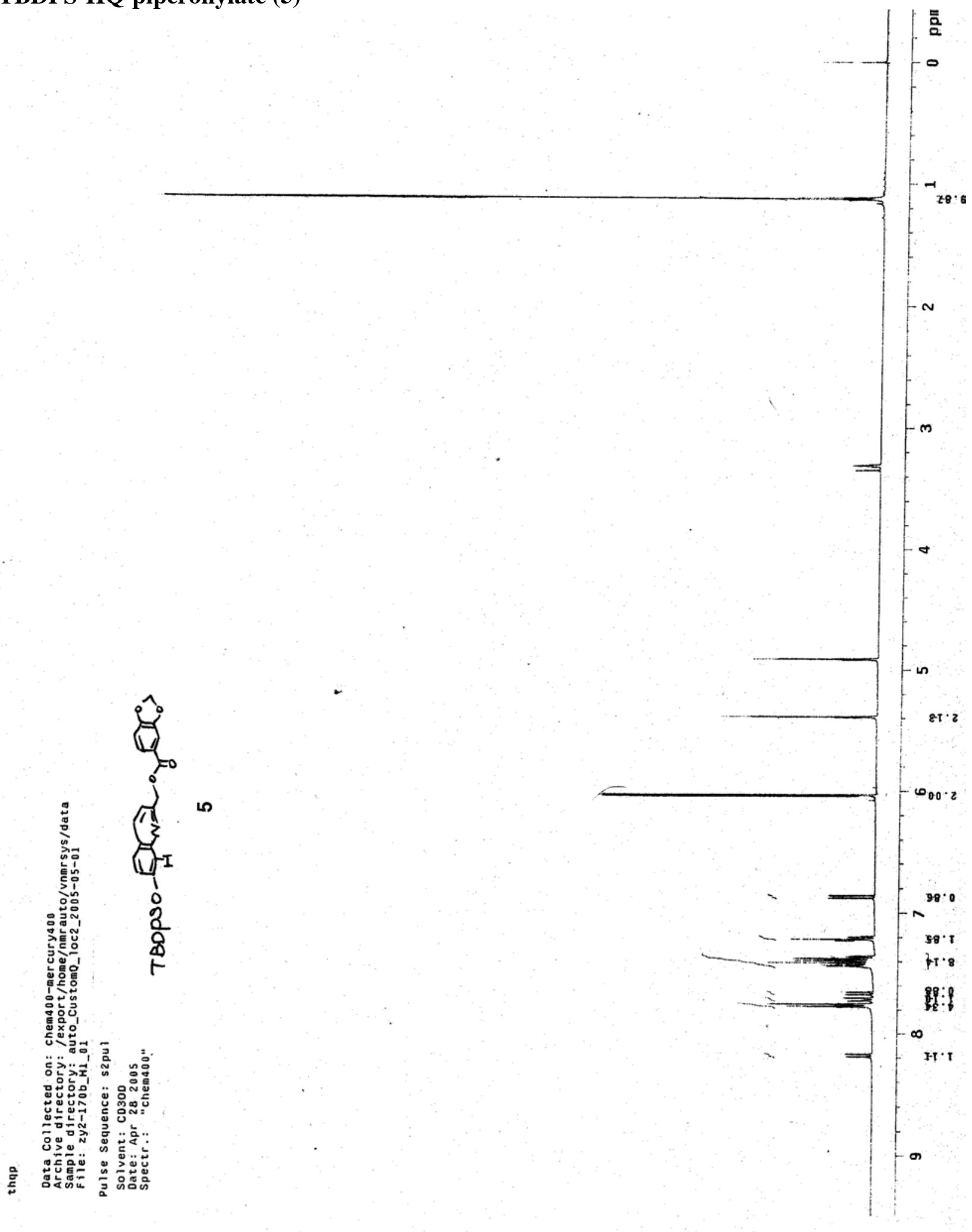









\section{BHQ-piperonylate (7)}
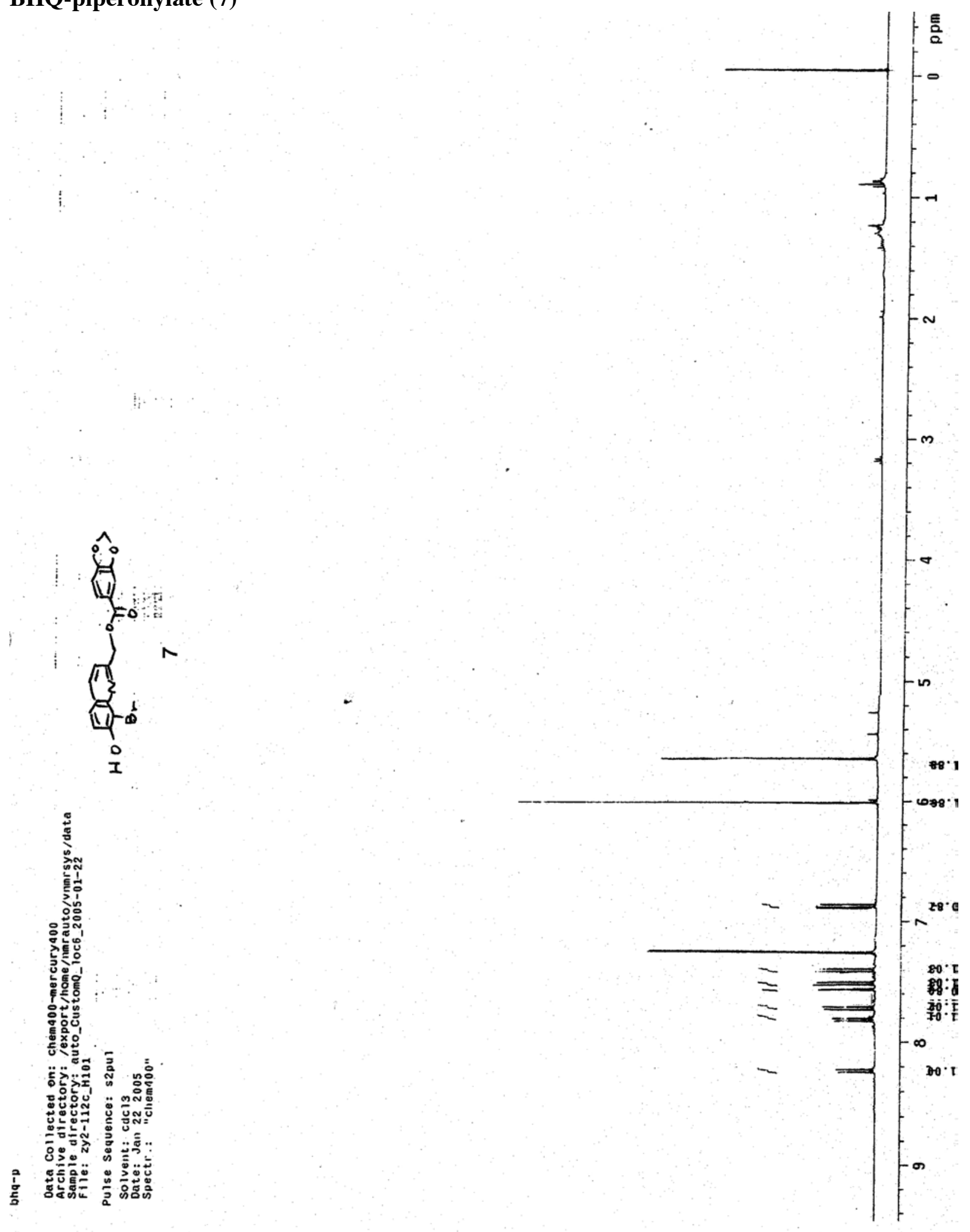
8-bromo-7-hydroxyquinaldine (9)




7-acetyl-8-bromoquinaldine (10)

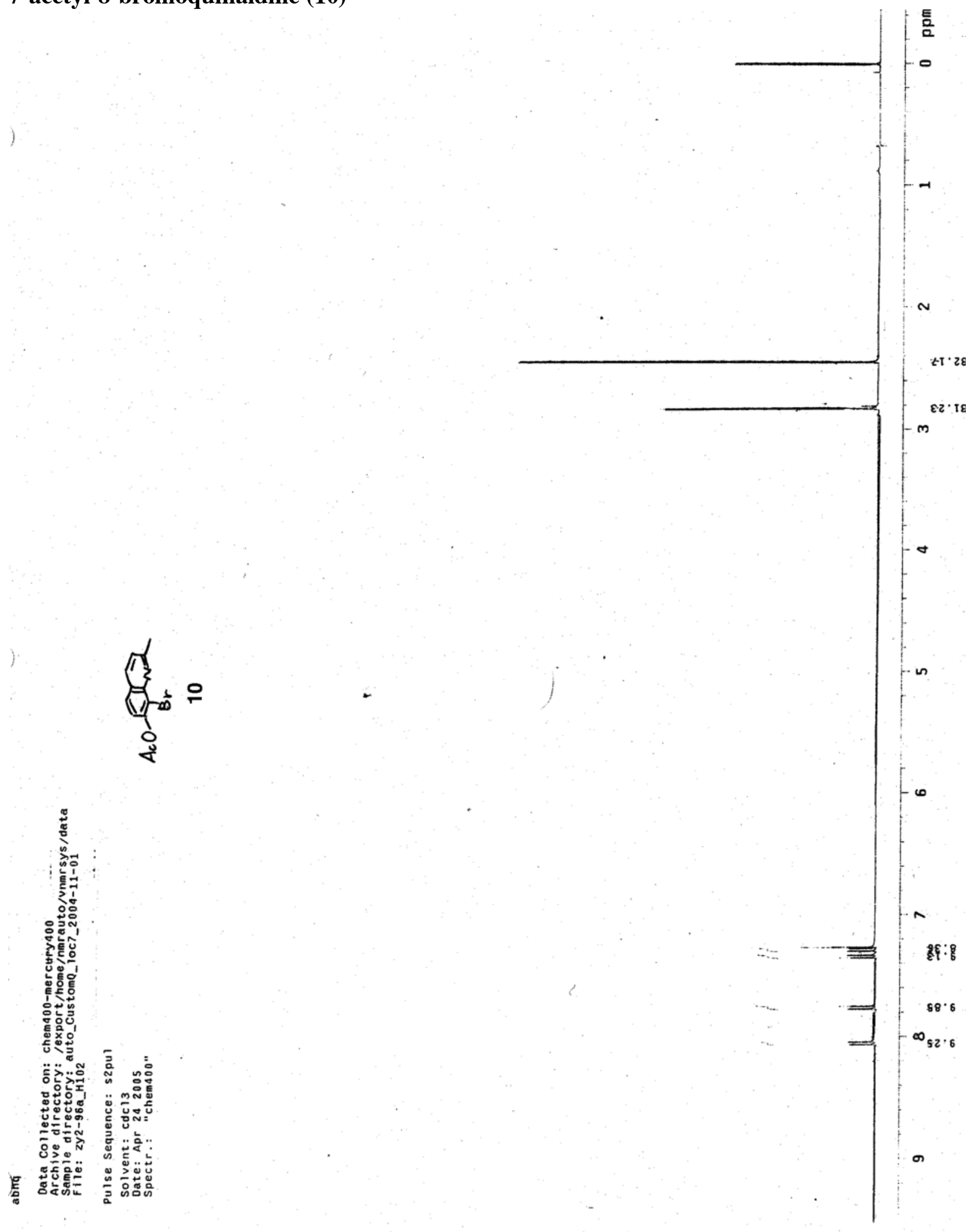


7-acetyl-8-bromoquinolin-2-yl formaldehyde (11)

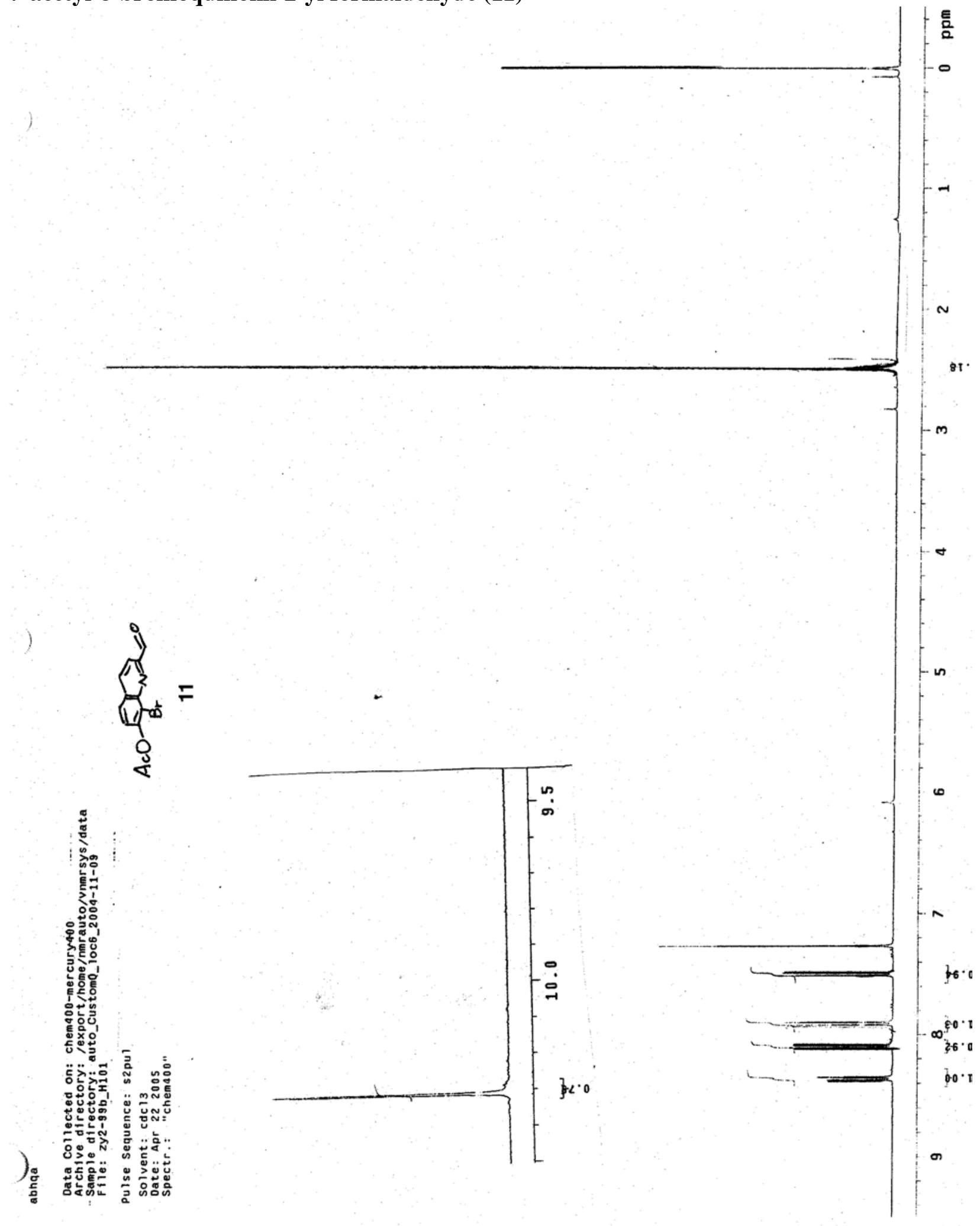


7-acetyl-8-bromo-2-formylquinolinyltosylhydrazone (12)

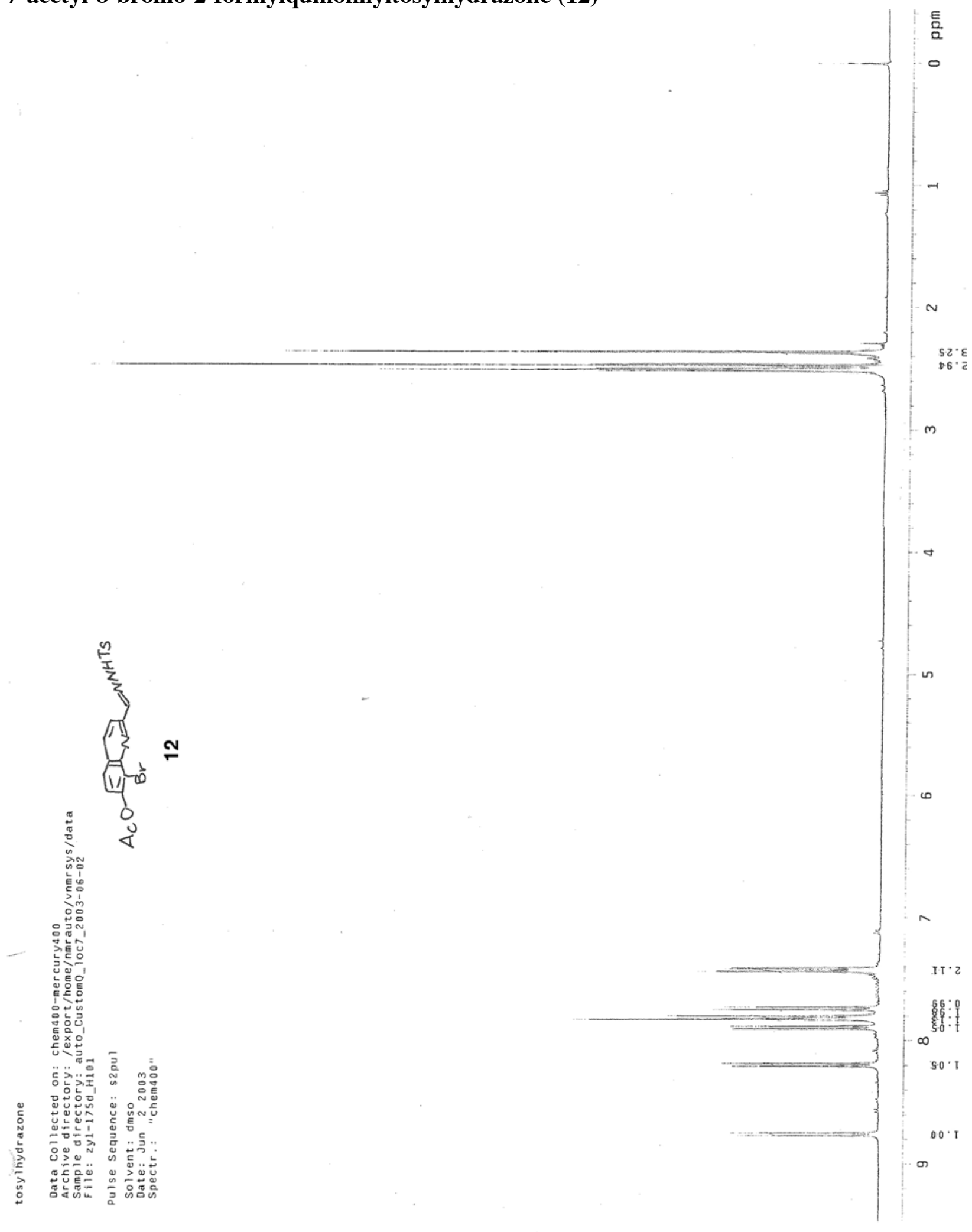


8-bromo-7-hydroxyquinolin-2-ylmethyl dimethylphosphate (13)

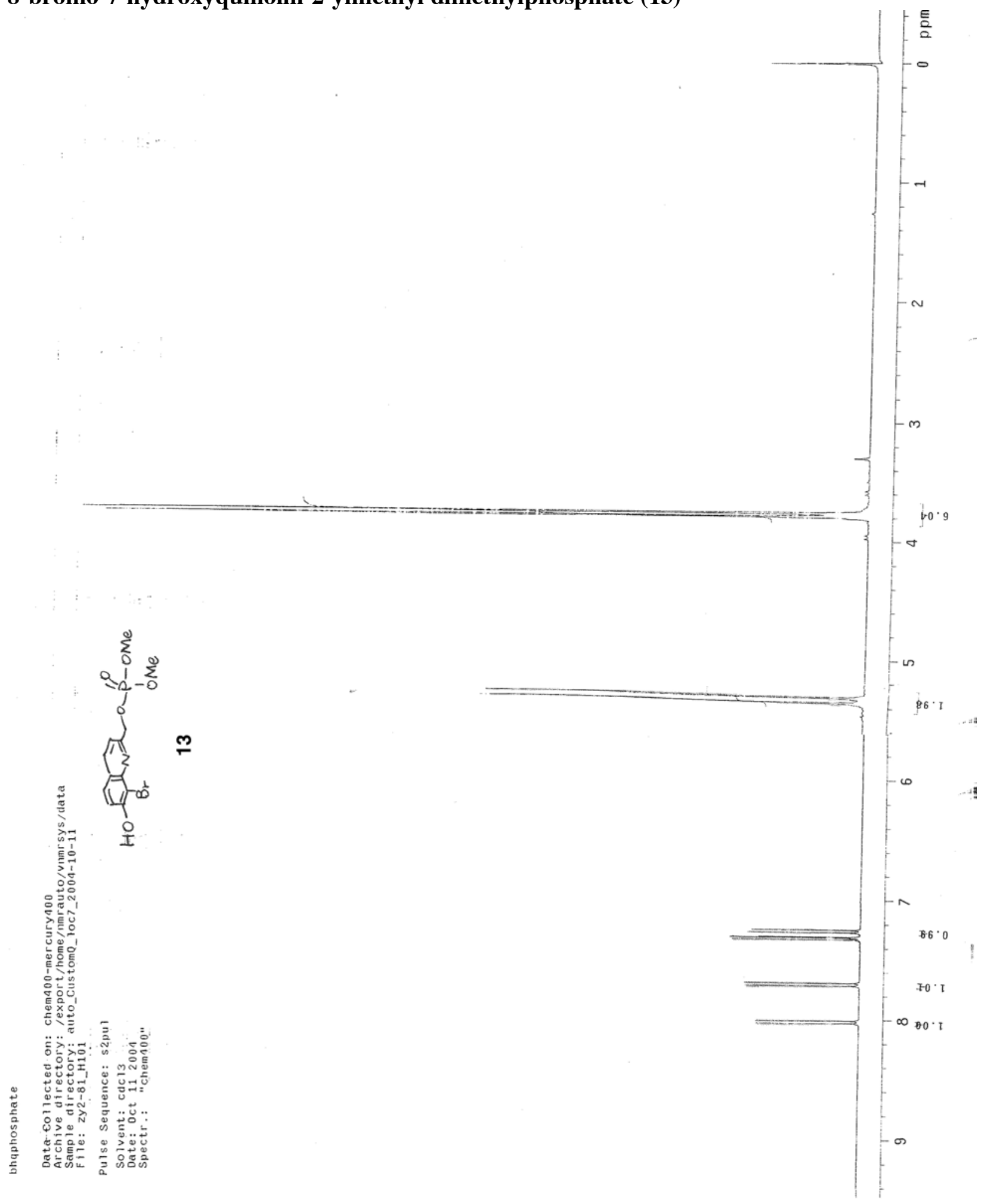




\section{BHQ-glycerol(OPh) (16)}

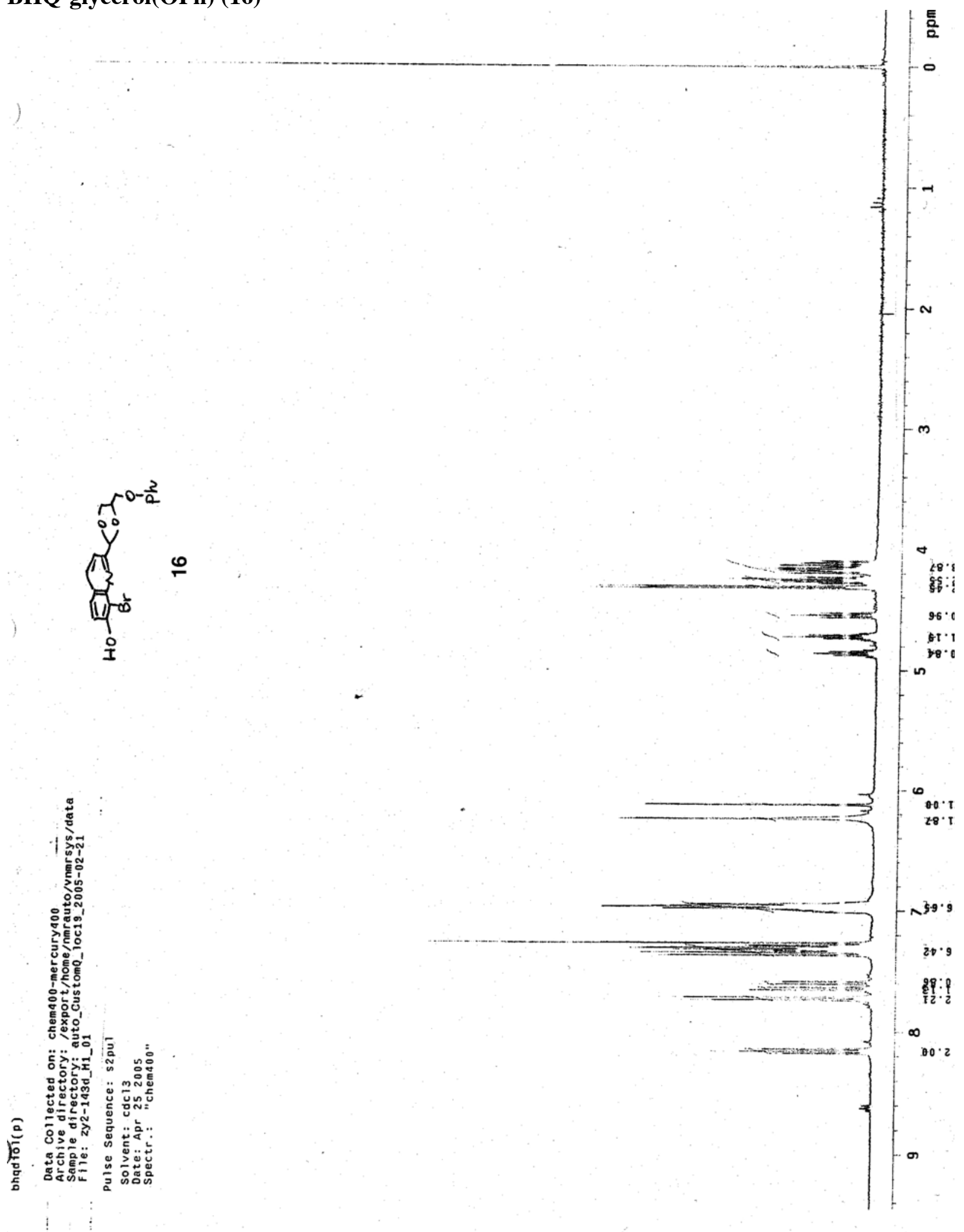




\section{BHQ-glycerol(SPh) (17)}



